\title{
PROBABILITY INEQUALITIES FOR SUMS OF WUOD RANDOM VARIABLES AND THEIR APPLICATIONS
}

\section{Lamei Chen, Kaiyong Wang, Miaomiao Gao and Yilun Dong}

Abstract. Let $n$ be a positive integer, $X_{1}, \ldots, X_{n}$ be real-valued random variables and $S_{n}=$ $\sum_{i=1}^{n} X_{i}$. When $X_{1}, \ldots, X_{n}$ are widely upper orthant dependent, some inequalities for the tail probability of $S_{n}$ have been given. The obtained results extend some existing results. As applications, the complete convergence of WOD random variables has been investigated.

Mathematics subject classification (2010): 60E15, 60F05.

Keywords and phrases: Widely upper orthant dependence, tail probability of sums, inequalities, complete convergence.

\section{REFERENCES}

[1] X. WANG, C. XU, T. HU, V. ANDREI, S. HU, On complete convergence for widely orthant dependent random variables and its applications in nonparametric regression models, TEST, 2014, 23(3): 607629.

[2] D. QiU, K. Chang, R. G. Antonini, On the strong rates of convergence for arrays of rowwisw negatively dependent random variables, Stochastic Analysis and Applications, 2011, 29(3): 375-385.

[3] N. Asadian, A. Bozorgnia, Rosenthal's Type Inequalities for Negatively Orthant Dependent Random Variables, Journal of the Iranian Statistical Society, 2006, 5(1): 69-75.

[4] H. W. Block, T. H. SAVITS, M. ShaKed Some concepts of negative dependence, Annals of Probability, 1982,10(3): 765-772.

[5] Y. Chen, A. Chen, K.W. NG, The strong law of large numbers for extended negatively dependent random variables, Journal of Applied Probability, 2010, 47(4): 908-922.

[6] M. GAO, K. WANG, L. Chen, Precise large deviations for widely orthant dependent random variables with different distributions, Journal of Inequalities and Applications, 2018, 2018: 1-21.

[7] D. QIU, P. CHEN, Complete and complete moment convergence for weighted sums of widely orthant dependent random variables, Acta Mathematica Sinica, 2014, 30(9): 1539-1548.

[8] F. CHENG, Y. WANG, J. YAN, The complete convergence of weighted product sums for NA sequences, Acta Mathematicae Applicatae Sinica, 2002, 25(4): 738-745.

[9] Y. WANG, Z. CUI, K. WANG, X. MA, Uniform asymptotics of the finite-time ruin probability for all times, Journal of Mathematical Analysis and Applications, 2012, 390(1): 208-223.

[10] X. HuAng, X. TAng, X. Deng, X. WANG, The large deviation for the least squares estimator of nonlinear regression model based on WOD errors, Journal of Inequalities and Applications, 2016, 2016: 1-11.

[11] N. Ebrahimi, M. Ghosh, Multivariate negative dependence, Communications in Statistics, 1981, 10(4): 307-337.

[12] P. Embrechts, C. Klüppelberg, T. Mikosch, Modelling extremal events for insurance and finance, 1997, Springer, Berlin.

[13] D. K. H. Fuk, S. V. NAGAev, Probability inequalities for sums of independent random variables, Theory of Probability \& Its Applications, 1971, 16(4): 643-660.

[14] K. WANG, Y. WANG, Q. GAO, Uniform asymptotics for the finite-time ruin probability of a new dependent risk model with a constant interest rate, Methodology \& Computing in Applied Probability, 2013, 15(1): 109-124.

[15] A. Gut, Complete convergence for arrays, Periodica Mathematica Hungarica, 1992, 25(1): 51-75. 
[16] X. WANG, S. HU, The consistency of the nearest neighbor estimator of the density function based on WOD samples, Journal of Mathematical Analysis and Applications, 2015, 429(1): 497-512.

[17] X. LI, X. LIU, W. YANG, S. Hu, The inverse moment for widely orthant dependent random variables, Journal of Inequalities and Applications, 2016, 2016: 1-11.

[18] V. M. Kruglov, A. I. Volodin, T. Hu, More on complete convergence for arrays, Statistics \& Probability Letters, 2006, 76(15): 1631-1640.

[19] E. L. Lehmann, Some concepts of dependence, Annals of Mathematical Statistics, 1966, 37(5): $1137-1153$.

[20] K. WANG, Y. YANG, J. Lin, Precise large deviations for widely orthant dependent random variables with dominatedly varying tails, Frontiers of Mathematics in China, 2012, 7(5): 919-932.

[21] L. LiU, Precise large deviations for dependent random variables with heavy tails, Statistics \& Probability Letters, 2009, 79(9): 1290-1298.

[22] Y. YAng, K. WANG, J. LiU, Asymptotics and uniform asymptotics for finite-time and infinite-time absolute ruin probabilities in a dependent compound renewal risk model, Journal of Mathematical Analysis and Applications, 2013, 398(1): 352-361.

[23] Y. YANG, K. WANG, J. LIU, Z. ZHANG, Asymptotics for a bidimensional risk model with two geometric Levy price processes, Journal of Industrial and Management Optimization, 2019, 15(2): 481-505.

[24] S.V. Nagaev, Some limit theorems for large deviations, Theory of Probability \& Its Applications, 1965, 10(10): 214-235.

[25] A. SHEN, Probability inequalities for END sequence and their applications, Journal of Inequalities and Application, 2011, 2011: 1-98.

[26] A. Shen, Y. Zhang, W. WANG, Complete convergence and complete moment convergence for extended negatively dependent random variables, Filomat, 2017, 31(5): 1381-1394.

[27] H. XIA, Y. WU, X. TAO, X. WANG, The consistency for the weighted estimator of nonparametric regression model based on widely orthant dependent errors, Probability in the Engineering and Informational Sciences, 2018, 32(3): 468-481.

[28] J. YAN, Complete convergence and complete moment convergence for maximal weighted sums of extended negatively dependent random variables, Acta Mathematica Sinica English, 2018, 2018: 116. 